\title{
Characterization and identification of Chinese historical rubbings preserved in Wuyuan Museum by Pyrolysis-Gas Chromatography/Mass Spectrometry
}

\author{
$\mathrm{Na} \mathrm{Yao}^{1}$, Xiangsheng Zhan ${ }^{2}$, Qinglin $\mathrm{Ma}^{3}$ and Shuya Wei ${ }^{i^{*}}$
}

\begin{abstract}
For conservation and restoration purposes, the precious historical rubbings preserved in Wuyuan Museum were studied by multi-analytical techniques including ${ }^{14} \mathrm{C}$ radiocarbon dating, scanning electron microscopy (SEM), Pyrolysis-Gas Chromatography/Mass Spectrometry (Py-GC/MS) and Herzberg staining method. The results showed that the Py-GC/MS method is an efficient method for identifying the raw materials of paper and ink in historical rubbings simultaneously. Through Py-GC/MS analysis, five types of constituents could be detected: (1) polycyclic aromatic hydrocarbons (PAHs) from soot; (2) compounds related to tar of conifer wood: retene and methyl dehydroabietate; (3) marker compounds from the whole egg; (4) additive compounds: menthol and curcumene, which were found for the first time in ink; (5) biochemical compounds from bark paper: $\beta$-amyrin and $a$-amyrin. Based on the above results, the ink type, binding media and additives in ink, as well as the fiber origin of the rubbing paper could be concluded. This study could not only provide scientific support for conservation and restoration of the historical rubbings preserved in Wuyuan museum, but also give method guidance for analysis of the uncharacterized rubbings from different origins.
\end{abstract}

Keywords: Rubbings, Pyrolysis-Gas Chromatography/Mass Spectrometry, Pine wood soot ink, Binding media, Additives, Fiber identification, Mulberry bark paper

\section{Introduction}

Rubbing is an ancient traditional Chinese skill to copy the characters and patterns on the stone carving or bronzes with handmade paper and ink [1]. The precious historical rubbings preserved in Wuyuan Museum of Jiangxi Province were produced from the stone carved the preface of Sanzang holy religion, which was written by Li Shi Min (Tai Zong Emperor of Tang Dynasty) and carved with Wang Xizhi's (famous calligrapher in the Eastern Jin Dynasty) calligraphy style in the third year of Tang Xianheng (A.D 672). For conservation and restoration

\footnotetext{
*Correspondence: sywei66@hotmail.com

${ }^{1}$ Institute of Cultural Heritage and History of Science \& Technology,

University of Science and Technology Beijing, Beijing 100083, PR China

Full list of author information is available at the end of the article
}

purposes, it is particularly important to characterize and identify the raw materials of paper and ink in the historical rubbings, mainly including the type of paper fiber, ink, binding media and additives in ink, etc.

China is the birthplace of traditional handmade paper. The most commonly used traditional Chinese handmade papers are made from fibers of ramie, bamboo, mulberry species, etc. The traditional method for identifying the raw materials of a paper is the Herzberg staining method [2-4], which is through observing the fiber morphological characteristic using microscopy to judge the fiber origins. In recent years, Pyrolysis-Gas Chromatography/ Mass Spectrometry (Py-GC/MS) and Pyrolysis-Gas twodimensional Chromatography-Mass Spectrometry (PyGCxGC/MS) have been introduced for the differentiation of East Asian handmade paper (kozo paper, mitsumata 
paper, gampi paper, mulberry bark paper, ramie paper and bamboo paper) according to the chemical markers of origin of the plant fibers, since each paper has characteristic pyrolysis fingerprints [5-8].

Chinese ink has a long history and a special importance in Chinese culture, which was made by mixing soot with pre-dissolved glue and additives, followed by kneading, pounding, molding and drying process [9]. The early Chinese ink was pine wood soot ink, which was obtained by the incomplete combustion of cedar, fir, hemlock, larch, pine and spruce, etc. The preparation process of pine wood soot ink was basically completed by the Han Dynasty (202 BC-AD 220) and developed in the later dynasties [10]. In the song dynasty and the later, the lamp soot ink was more widely in use than the pine wood soot ink. The lamp soot was mainly obtained from the incomplete combustion of vegetable oil and animal oil [11]. The most frequently used binding media are animal glue, vegetable glue and egg glair/egg york, etc. In addition, the commonly used additives in ink mainly contained camphor, borneol and lacquer, etc. [12]. Ink was usually characterized through the morphology and size of the soot particles by using scanning electron microscopy (SEM) [13] and transmission electron microscope (TEM) [14]. These methods cannot identify the binding media and additives in ink. Py-GC/MS was applied to characterize Chinese ink by Wei et al. in 2011, through the detection of main chemical compounds in soot including phenanthrene, fluoranthene, pyrene, triphenylene and benzofluoranthene and their relative amount, pine wood soot ink and lamp soot ink can be differentiated [15]. Subsequently, this method and the criteria were used for the identification of the archaeological ink stick in the Eastern Jin Dynasty (317-420 AD), which was identified as pine wood soot ink; Meanwhile, animal glue was found as binding media; borneol and cedar oil were detected as additives in the ink stick [16]. Recently, pine wood soot was also identified in the ink as early as the Han Dynasty (202 BC-AD 220) by Py-GC/MS [17].

In summary, from literature it can be seen that $\mathrm{Py}-\mathrm{GC} /$ MS was successfully used for the identification of paper fibers, soot, binding media, and additives in Chinese ink, respectively. The rubbings are made of handmade paper covered with Chinese ink, so thus Py-GC/MS was applied to characterize both the paper and the ink of the rubbings simultaneously, hopefully the fiber origin of the paper, the soot and additives, as well as the binding media used in ink could be identified in one analysis. In addition, ${ }^{14} \mathrm{C}$ radiocarbon dating, SEM and Herzberg staining method were also applied for complementary information. This paper demonstrates the methodology chosen could obtain maximum information with a small amount of sample, which is in accordance with the special requirement of a precious sample, such as historical rubbings. Furthermore, the results obtained through this study could not only provide evidence for its value, but also supply scientific support for its conservation and restoration.

\section{Experimental \\ Materials \\ Rubbing sample}

A sample was taken from the page fringe of one historical rubbing (Fig. 1) of the Sanzang Holy Preface for analyses, which were preserved in the Wuyuan Museum of Jiangxi Province, China. It was observed that rubbing paper has smooth texture, clear and regular laid lines, characterized by 24 laid lines in $3 \mathrm{~cm}$, showing that the rubbing paper was made on a moveable sieve constructed of bamboo.

\section{Modern reference paper samples}

Typical traditional Chinese handmade papers made from mulberry bark (Morus alba L.), paper mulberry (Broussonetia papyrifera) and mixed fibers of Pteroceltis tatarinowii Maxim bark and Oryza sativa L. (Xuan paper) were investigated. Mulberry bark paper was from Qianshan county in Anhui province, paper made of paper mulberry was from Zhenfeng county in Guizhou province, while Xuan paper was from Jingxian county in Anhui province, respectively.

\section{Techniques and methods \\ ${ }^{14} \mathrm{C}$ radiocarbon dating}

The radiocarbon dating was conducted by the quaternary dating laboratory of the accelerator mass spectrometry laboratory in Peking University.

\section{SEM}

TESCAN VEGA 3 XMU scanning microscope. For this analysis, firstly the historical rubbing sample was coated with $\mathrm{Au}$, then carbon adhesive tape was used to hold the sample for SEM observation. The SEM observation was conducted with $15 \mathrm{kV}$, WD $9 \mathrm{~mm}$.

\section{Py-GC/MS}

It was performed using a vertical micro furnace-type pyrolyzer PY-3030D (Frontier Lab, Japan) directly connected to the injection port of a Shimadzu QP2010Ultra gas chromatograph mass spectrometer (Shimadzu, Japan). The sample was placed in a stainless steel sample cup. Reference paper used in this work for the development of the analytical method was $2.5 \mathrm{~mm} \times 2.5 \mathrm{~mm}$ size. The sample cup was placed on top of the pyrolyzer at near ambient temperature. The sample cup was introduced into the furnace at $600{ }^{\circ} \mathrm{C}$, and then the temperature program of the gas chromatograph oven was 

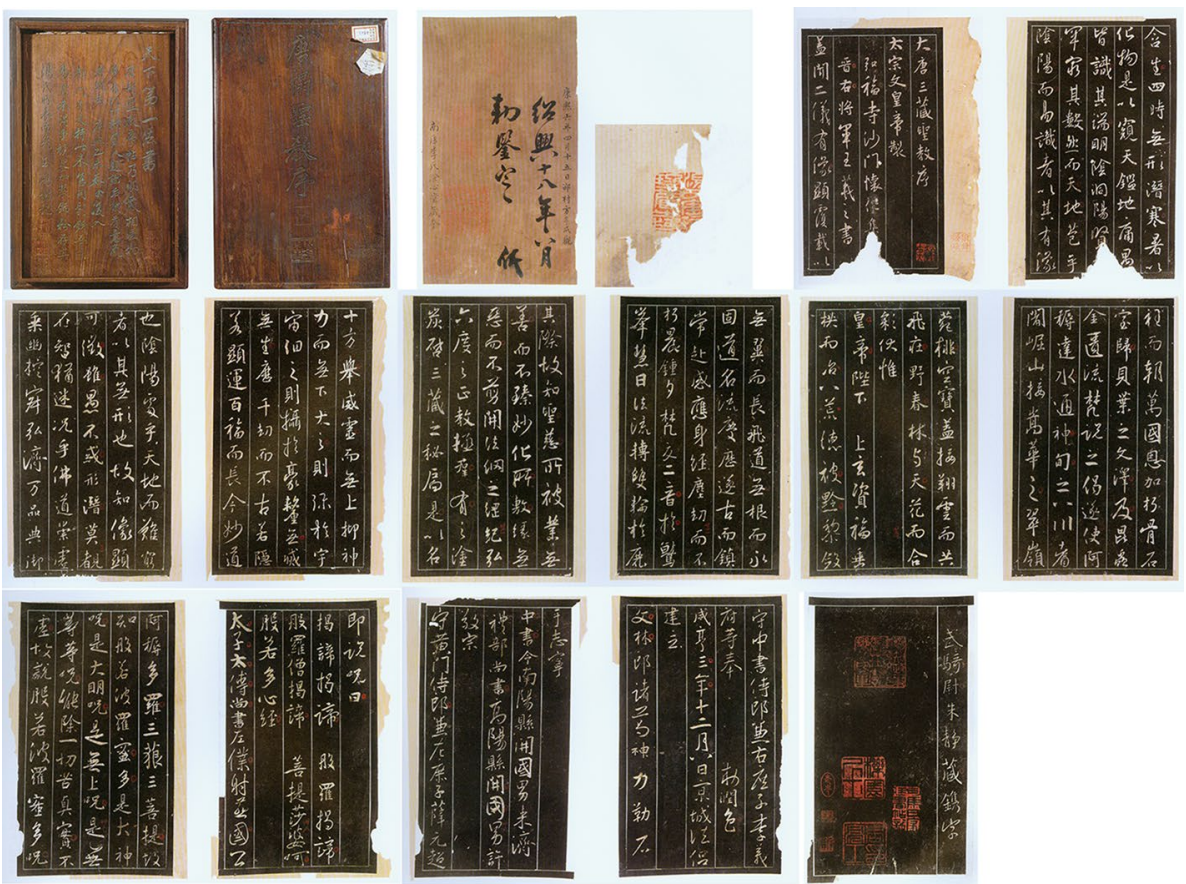

Fig. 1 The historical rubbings in Wuyun Musum of Jiangxi Province

started. The Py-GC interface was held at $320^{\circ} \mathrm{C}$. Chromatographic separation was carried out on an UA +-5 Frontier Lab 5\%-dimethyl diphenyl polysiloxane column (30 m length, $0.25 \mathrm{~mm}$ inner diameter and coated with a $0.25-\mu \mathrm{m}$ film thickness). The oven temperature was initially held $3 \mathrm{~min}$ at $40{ }^{\circ} \mathrm{C}$, and then ramped at $5{ }^{\circ} \mathrm{C} \mathrm{min}{ }^{-1}$ to $325^{\circ} \mathrm{C}$, where it was held for $5 \mathrm{~min}$. The total duration of GC analysis was $65 \mathrm{~min}$. The helium carrier gas, was used in the linear velocity mode $\left(1 \mathrm{~mL} \mathrm{~min}^{-1}\right)$. The injector was held at $280{ }^{\circ} \mathrm{C}$ and used in split mode (1:10 of the total flow). A scan range from 50 to 750 was used in mass spectrometer, using electron ionization at $70 \mathrm{eV}$. The interface was kept at $280^{\circ} \mathrm{C}$ and the MS source at $200{ }^{\circ} \mathrm{C}$. Identifications were achieved on the basis of EI mass spectra by interpretation of the main fragmentations and using the NIST14 and NIST14s MS library. The same amount of sample was pyrolysed in three replicates, and the variability between replicate pyrograms was minimal. Three parallel samples were analyzed the peak area of the compounds and the standard deviations were calculated by using three groups of data. A blank run (sometimes two or three) was inserted between each pair of actual analyses to be able to rule out such influences.

\section{Herzberg staining method}

Leica microsystems made in Germany (DM2700P) were used to observe the morphological characteristic of the sample fibers after the iodine-zinc chloride reagents. The preparation method of the iodine-zinc chloride reagents refers to Li's literature [18].

\section{Results and discussions \\ ${ }^{14} \mathrm{C}$ radiocarbon dating}

The historical rubbings preserved in Wuyuan museum were produced from the stone carved with the preface of Sanzang holy religion, which was written by the Emperor -Li Shi Min in the Tang dynasty, so the earliest date of these rubbings was possibly in Tang dynasty. For confirmation of the time when the rubbings were produced, the radiocarbon dating analysis was conducted by the accelerator mass spectrometry laboratory in Peking University. A piece of paper was taken from the rubbings as a sample. The analysis result indicates that the date of the rubbings is $1680 \pm 30$ A.D, which is the early year of the Qing dynasty in China.

\section{Characterization of the rubbing sample by using SEM}

The morphological characteristic of ink on the historical rubbing sample was observed by scanning electron microscopy (SEM) at a magnification of $20,000 \times$. The result is shown in Fig. 2. As can be seen, the ink particles were shaped like balls and nonuniform in size. This feature is consistent with the characteristic of wood soot ink, which gives a clue that the ink 


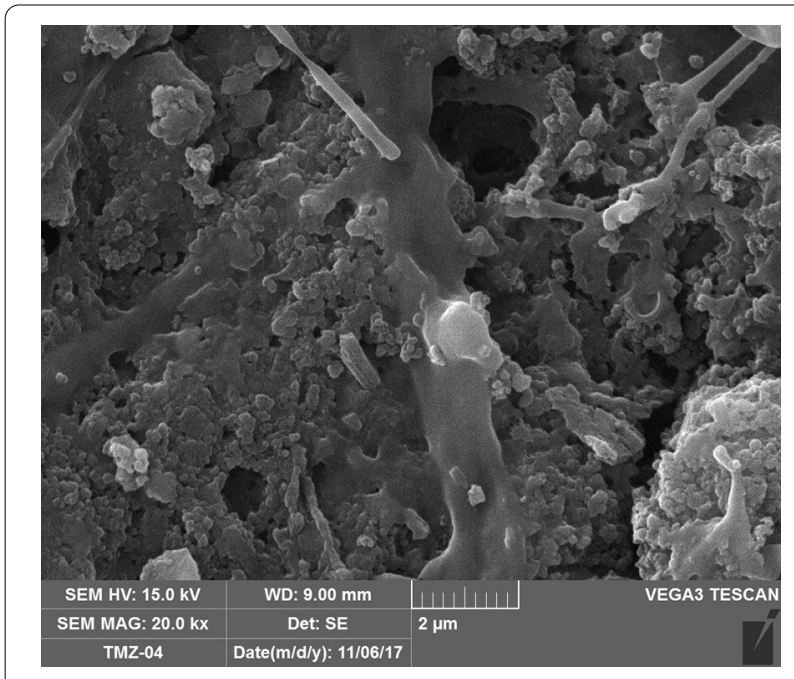

Fig. 2 SEM image of ink in the rubbing sample $\times 20,000$

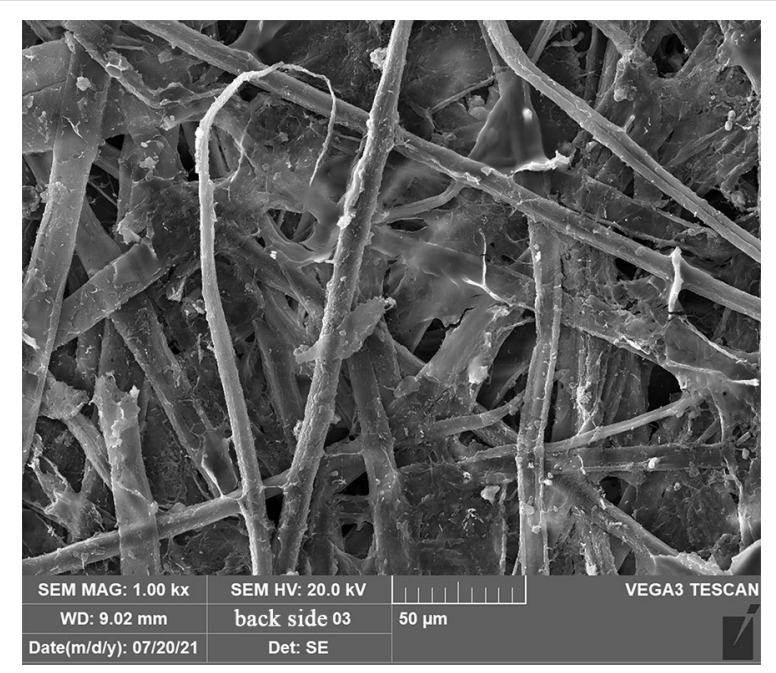

Fig. 3 SEM image of paper fibers on the back side of rubbing sample $\times 1000$

used in rubbings is probably wood soot ink [14]. As shown in Fig. 3, scanning electron microscope (SEM) was also used to analyze the paper fibers on the back side of historical rubbing sample. It can be seen that a small amount of ink particles was penetrated into the back side of the rubbing sample, most fibers are long and thick, and some fiber disconnection was detected, which indicates the presence of degradation of historical rubbing. Through fiber features observation, the type of paper fibers could not be determined by using
SEM. Further investigation by $\mathrm{Py}-\mathrm{GC} / \mathrm{MS}$ was conducted, which is stated in the following subsection.

\section{Characterization of the historical rubbings by using Py-GC/MS}

Py-GC/MS was utilized to analyze the historical rubbing sample, the chromatogram obtained is presented in Fig. 4, while the primary compounds identified are listed in Table 1. Five types of substances were detected in the historical rubbing sample by using Py-GC/MS analysis: (1) a series of polycyclic aromatic hydrocarbons (PAHs) could be identified as the following compounds: anthracene, fluoranthene, pyrene, triphenylene, benzo[k] fluoranthene and benzo[ghi]perylene, labeled as S1, S2, S3, S4, S5, and S6 in Fig. 4 respectively. The above PAHs are from soot, in which the relative high contents of S5 and $S 6$ could be used as an indication of the presence of certain soot [15]; (2) a small number of compounds including retene (as shown in Fig. 4, labeled as T1) and methyl dehydroabietate (as shown in Fig. 4, labeled as T2) were detected, the chemical structures of the two compounds were shown in Fig. 5. T1 and T2 could be from tar of conifer wood according to the literature [16], while T2 could be also from a resin origin; (3) in Fig. 4, G1, G2 and G3 represent 3-methyl-1H-pyrrole, indole, and 3-methyl-indole respectively. Indole and methyl indole are the main pyrolysis products of tryptophan, which are marker compounds of egg glair $[19,20]$. In addition, high contents of $\mathrm{C}_{16}$ and $\mathrm{C}_{18}$ fatty acids, as well as cholesta3,5-diene (labeled as G4 in Fig. 4) were also detected, indicating the presence of egg yolk [19]; (4) compounds of menthol and curcumene were detected in the rubbings (labeled as E1 and E2 in Fig. 4), the chemical structures were shown in Fig. 5. Menthol is the main chemical component of peppermint oil, which is obtained from the roots, stems and leaves of peppermint by steam distillation. It is an important flavor additive [21], which is the first time found in ancient ink. Curcumene is the main chemical component of the essential oil of turmeric herbs, which has antibacterial, antitumor and antioxidant effects [22, 23]; (5) furthermore, furan structure (as shown in Fig. 4, labeled as 3), ketones (as shown in Fig. 4, labeled as 2 and 5), carbohydrate (as shown in Fig. 4, labeled as 10) and a series of compounds characterized by a base peak at $218 \mathrm{~m} / \mathrm{z}$, corresponding to the beta-amyrin (as shown in Fig. 4, labeled as 13) and alphaamyrin (as shown in Fig. 4, labeled as 14) were noticed, which are the biochemical marker compounds of the papers made of mulberry bark (Morus alba L.) or paper mulberry (Broussonetia papyrifera) [8].

The historical rubbing sample was also studied with thermal assistant methylation Py-GC/MS technique analysis. The methylating reagent used is 


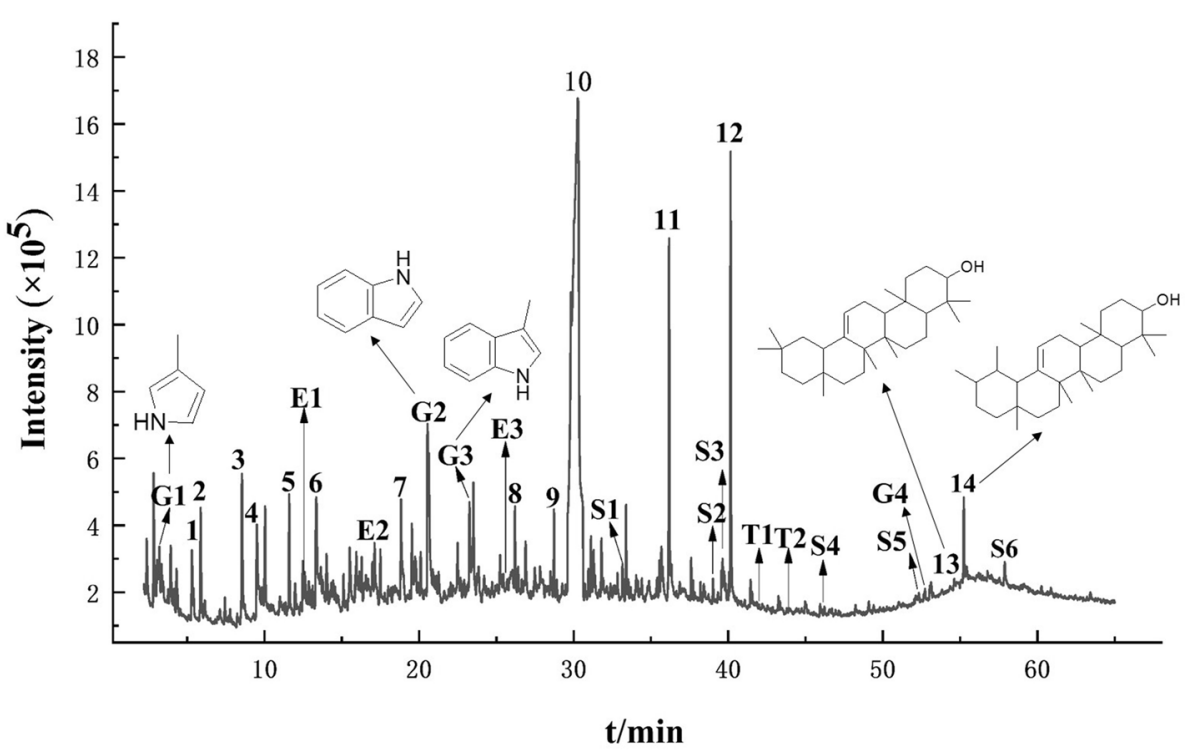

Fig. 4 Total ion current chromatogram obtained from the pyrolysis of the historical rubbing sample, the peak numbers correspond to the numbers in Table 1

tetramethylammonium hydroxide (TMAH). The obvious difference is that a series of fatty acid methyl esters are detected. However, the characteristic compounds of fibers and soot could not be detected.

In order to compare the Py-GC/MS analysis results of the rubbings to modern reference samples, the chromatograms of modern bark papers (Morus alba paper, Broussonetia papyrifera paper, Xuan paper) obtained by Py-GC/MS analysis are shown in Fig. 6. It can be seen that $\beta$-amyrin, $\alpha$-amyrin, and stigmastan- 3,5 -diene were the characteristic compounds in papers made of mulberry bark (Morus alba) and paper mulberry (Broussonetia papyrifera), but they were not existent in Xuan paper made of the mixed fibers of Pteroceltis tatarinowii Maxim bark and Oryza sativa L. The detection of $\beta$-amyrin and $\alpha$-amyrin in rubbing sample indicates that the paper of rubbings is made of mulberry bark fibers or paper mulberry fibers, not Xuan paper.

In order to see the relative contents of the PAHs, analyses by Py-GC/MS with selection mode (SIM) for S1-S6 were conducted. The chromatogram obtained is depicted in Fig. 7. For comparison, the relative contents of the PAHs of the reference materials of the pine wood soot ink (PM), lamp soot ink (LM) and carbon black (CB), which were published earlier [15], as well as the PAHs results of the historical rubbings (RM) are listed in Table 2. The relative content of benzo[k]fluoranthene (S5) in PM (20.2\%, peak area) is higher than in LM (12.5\%, peak area), while CB has no triphenylene
(S4). The relative content of benzo[k]fluoranthene in the rubbings is $22.2 \% \pm 1.8$ (peak area), and the triphenylene is $11.3 \% \pm 1.3$ (peak area), which is in accordance with that of pine wood soot ink (PM). In addition, combining the detection of marker compounds of retene and methyl dehydroabietate, pine wood soot ink used for the rubbings can be concluded.

In summary, the ink used for the rubbings is pine wood soot ink, with whole egg (egg glair and egg yolk) as binding media, while peppermint oil and turmeric herbs oil are possibly used as additives in ink. No surprise that the finding of pine wood soot ink in the rubbings, since its use can date back as early as the Han Dynasty (202 BC-AD 220) [10], which was continuously in use in the following Dynasties. Animal glue is the common binding media used in Chinese ink [11]. However, in this case, the whole egg was found as binding media. Interestingly, it is the first time that peppermint oil and turmeric herbs oil were found as additives in ink. The most commonly used additives in ink mainly contained camphor, cedar oil and borneol, etc. [12].

Using Py-GC/MS method, the paper in rubbings is paper made of mulberry bark fibers or paper mulberry fibers. It is difficult to differentiate mulberry bark paper from paper made of paper mulberry by $\mathrm{Py}-\mathrm{GC} / \mathrm{MS}$ analysis, since they both belong to the Moraceae family and have similar pyrolysis characteristic compounds. In order for the unambiguous confirmation of the fibers in rubbings, Herzberg staining method was conducted in the following. 
Table 1 List of compounds of the rubbing paper with retention time (RT), expected main fragment ions, most likely attribution of the products, and relative content

\begin{tabular}{|c|c|c|c|c|}
\hline No & $\mathrm{RT}(\mathrm{min})$ & Main ions & Identified compounds & Area $(\%) \pm S D$ \\
\hline G1 & 3.35 & $53,(80)$ & 1H-Pyrrole, 3-methyl- & $0.84 \pm 0.16$ \\
\hline 1 & 5.32 & $51,78,(104)$ & Styrene & $1.15 \pm 0.14$ \\
\hline 2 & 5.85 & $53,(67), 96$ & 2-Cyclopenten-1-one, 2-methyl- & $1.6 \pm 0.35$ \\
\hline 3 & 8.57 & $53,67,(96)$ & 2,4-Dimethylfuran & $2.49 \pm 0.58$ \\
\hline 4 & 9.51 & 66, (94) & Phenol & $1.8 \pm 0.18$ \\
\hline 5 & 11.61 & $(67), 95,110$ & 2-Cyclopenten-1-one, 2,3-Dimethyl- & $1.26 \pm 0.35$ \\
\hline E1 & 12.61 & 69, (109), 119, 134 & Cis-p-mentha-1 (7),8-dien-2-ol & $0.82 \pm 0.08$ \\
\hline 6 & 13.35 & 79, (108) & 3-Methyl-Phenol & $2.73 \pm 0.61$ \\
\hline E2 & 16.98 & $(71), 81,95,123,138$ & Menthol & $0.61 \pm 0.22$ \\
\hline 7 & 18.86 & $(91), 131$ & Benzenepropanenitrile & $1.54 \pm 0.57$ \\
\hline G2 & 20.57 & $63,90,(117)$ & Indole & $2.38 \pm 0.56$ \\
\hline G3 & 23.29 & $77,103,(130)$ & 3-Methyl-Indole & $1.8 \pm 0.44$ \\
\hline E3 & 25.58 & 105, (119), 132, 202 & Curcumene & $0.4 \pm 0.11$ \\
\hline 8 & 26.2 & $(55), 69,83,97,111$ & 1-Pentadecene & $1.01 \pm 0.29$ \\
\hline 9 & 28.73 & $(55), 69,83,97,111$ & 1-Heptadecene & $0.89 \pm 0.31$ \\
\hline 10 & 30.29 & $(60), 73$ & 1,6-Anhydro-bete-D-Glucopyranose & $21.5 \pm 6.94$ \\
\hline S1 & 33.2 & $76,152,(178)$ & Anthracene & $0.38 \pm 0.12$ \\
\hline 11 & 36.2 & $43,(73), 129,256$ & n-Hexadecanoic acid & $4.09 \pm 0.7$ \\
\hline S2 & 39.01 & $101,(202)$ & Fluoranthene & $0.24 \pm 0.01$ \\
\hline S3 & 40.05 & $101,(202)$ & Pyrene & $0.26 \pm 0.08$ \\
\hline 12 & 40.18 & $43,(73), 129,284$ & Octadecanoic acid & $4.96 \pm 1.29$ \\
\hline $\mathrm{T} 1$ & 41.9 & 189, 204, (219), 234 & Retene & $0.22 \pm 0.07$ \\
\hline $\mathrm{T} 2$ & 44.02 & (239), 299, 314 & Methyl dehydroabietate & $0.12 \pm 0.03$ \\
\hline S4 & 46.07 & $101,113,(228)$ & Triphenylene & $0.15 \pm 0.04$ \\
\hline S5 & 52.33 & $113,125,(252)$ & Benzo[k]fluoranthene & $0.19 \pm 0.04$ \\
\hline G4 & 52.77 & $57,81,147,353,(368)$ & Cholesta-3,5-diene & $0.21 \pm 0.05$ \\
\hline 13 & 54.66 & $95,135,189,203,(218)$ & Beta-amyrin & $0.18 \pm 0.04$ \\
\hline 14 & 55.3 & $95,135,189,203,(218)$ & Alpha-amyrin & $1.06 \pm 0.31$ \\
\hline s6 & 57.87 & $138,(276)$ & Benzo[ghi]perylene & $0.29 \pm 0.02$ \\
\hline
\end{tabular}

The value of the main ions in parenthesis is the base peak

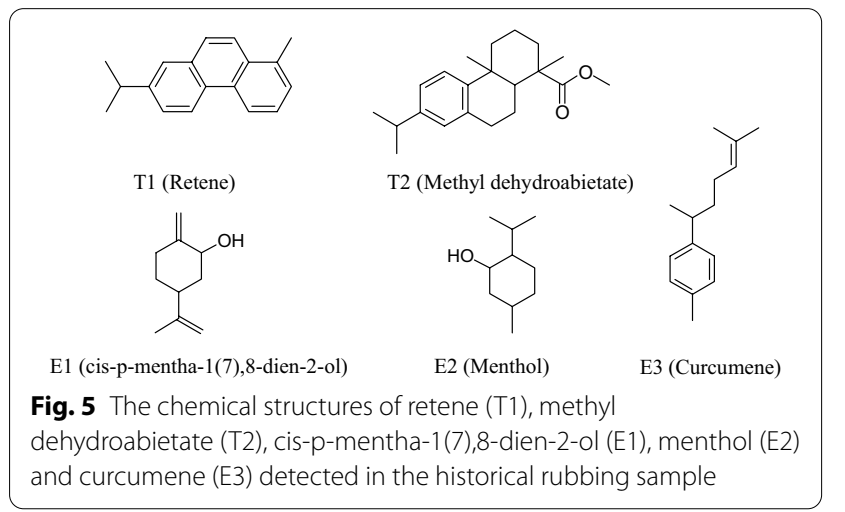

\section{Fiber identification using Herzberg staining method}

Modern reference papers made of mulberry (Morus sp.) and paper mulberry (Broussonetia sp.) were analyzed under the microscope after Herzberg staining method treated, respectively. As shown in Figs. 8 and 9, the morphological characteristics of the two kinds of fibers are similar, mainly including: (1) the reddish-brown color of the fibers; (2) obvious transverse nodal lines, and most of the nodal spacing is larger than the fiber diameter; (3) as well as thin and transparent layer (Jiaoyi) on the outer wall of fiber. However, a certain amount of crystals (labeled in Fig. 8) were observed only in paper mulberry (Broussonetia sp.) fiber, but not in mulberry fiber, which could be used to differentiate mulberry bark paper from paper made of paper mulberry.

According to the Herzberg staining method, the fibers taken from the historical rubbing sample were examined under the microscope, the result is shown in Fig. 10. The morphological characteristic of the rubbing paper fiber was not so obvious due to the presence of ink, but by 


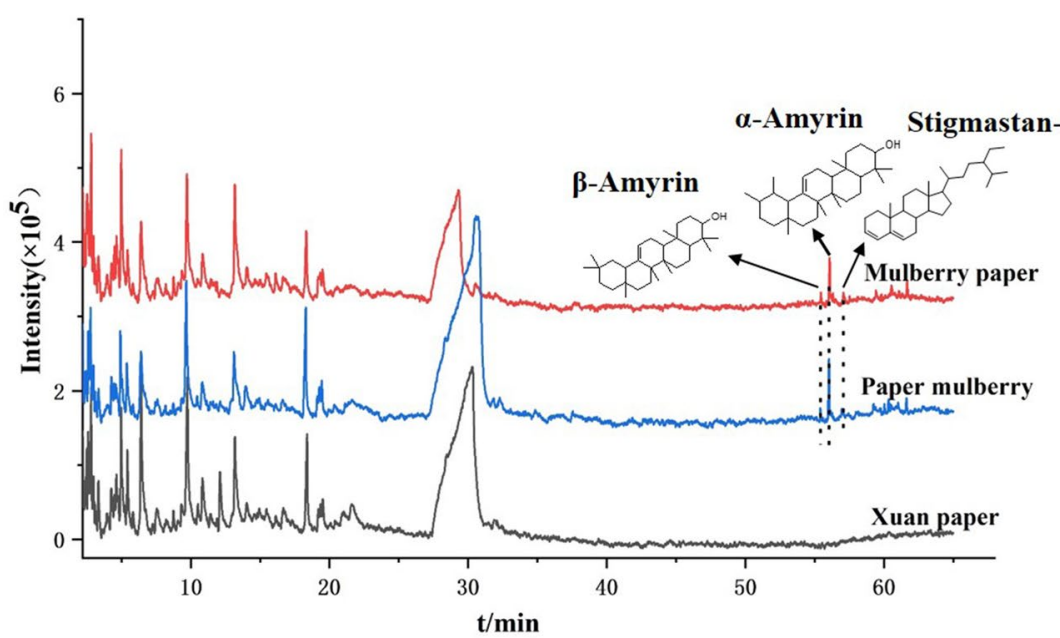

Fig. 6 Total ion current chromatograms obtained from the pyrolysis of the reference mulberry bark paper, paper mulberry paper and Xuan paper

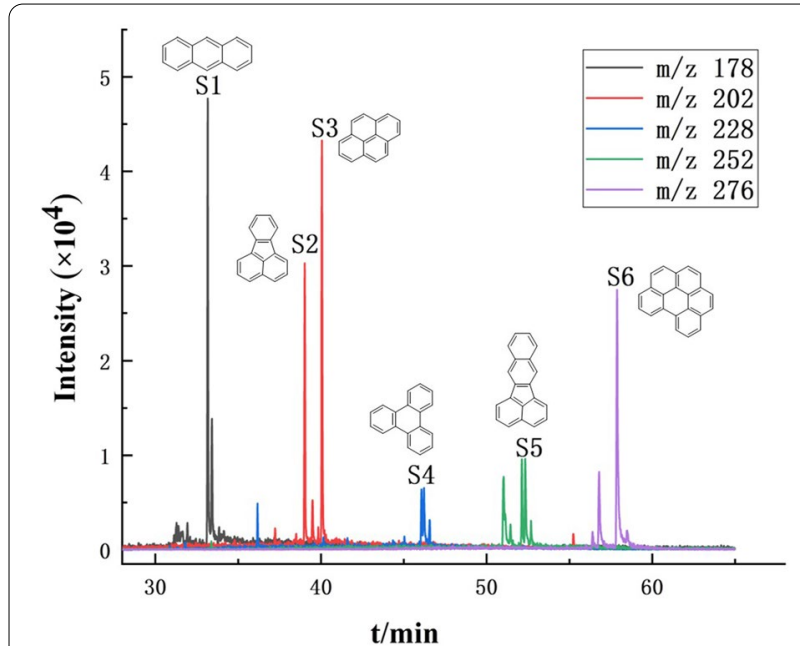

Fig. 7 The select ions chromatograms (SIM) of ink in historical rubbing sample by using Py-GC/MS

Table 2 The relative contents of the main PAHs in the modern pine wood ink (PM), lamp soot ink (LM), carbon black (CB) and the ink used in the historical rubbings (RM) by Py-GC/MS analysis

\begin{tabular}{llrrr}
\hline PAHs & \multicolumn{4}{l}{ Sample } \\
\cline { 2 - 5 } & PM & LM & CB & RM \pm SD \\
\hline S1 area(\%) (m/z 178) & 13.9 & 14.5 & 71.4 & $27.0 \pm 1.7$ \\
S2 area(\%) (m/z 202) & 27 & 34.3 & 9.8 & $17.8 \pm 1.5$ \\
S3 area(\%) (m/z 202) & 28.9 & 34.7 & 9.1 & $20.1 \pm 1.8$ \\
S4 area(\%) (m/z 228) & 10 & 3.8 & 0.0 & $11.3 \pm 1.3$ \\
S5/S6 area(\%) (m/z 252) & 20.2 & 12.5 & 9.5 & $22.2 \pm 1.8$ \\
\hline
\end{tabular}

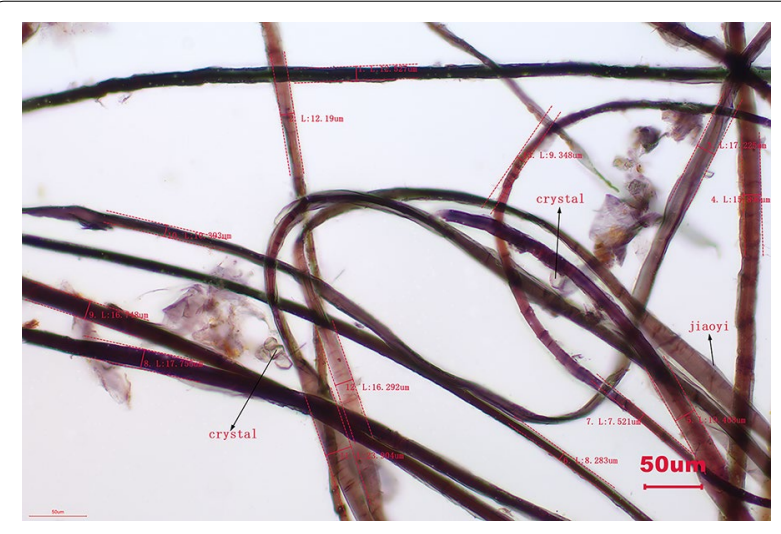

Fig. 8 The fiber morphology of a modern handmade paper made of paper mulberry $\times 20$

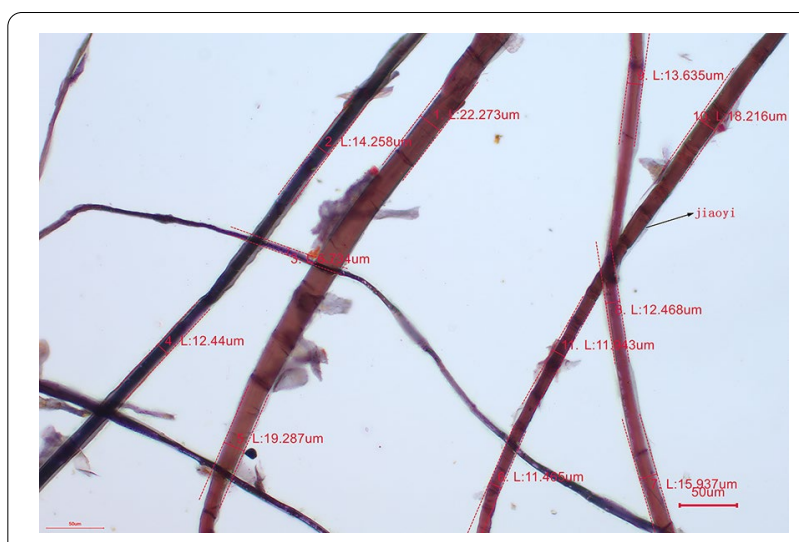

Fig. 9 The fiber morphology of a modern handmade paper made of mulberry $\times 20$ 
comparing with that of modern reference papers made of mulberry bark and paper mulberry, similar fiber features could be observed. The most importance is that no crystals were found in the rubbing paper fibers, indicating the rubbing paper was made of mulberry bark fibers.

According to the Book of the Later Han (Cai Lun's biography), the raw materials for papermaking included ramie rags, fishing nets, and paper mulberry during the Eastern Han Dynasty. During the Tang Dynasty, paper mulberry bark, mulberry bark, and rattan bark were the primary sources for papermaking [24]. In the later dynasties and even now, mulberry bark paper is still in use.

\section{Conclusions}

In this study, multi-analytical techniques including ${ }^{14} \mathrm{C}$ radiocarbon dating, Pyrolysis Gas Chromatography/ Mass Spectrometry (Py-GC/MS), scanning electron microscope (SEM), as well as Herzberg staining method were utilized for the characterization and identification of the precious historical rubbings collected in Wuyuan Museum of Jiangxi Province, China. The research demonstrated that the Py-GC/MS method is an efficient method for identifying the raw materials of paper and ink in historical rubbings simultaneously. The rubbing fiber source could be identified as mulberry bark fibers or paper mulberry fibers. Combining with the analysis results by Herzberg staining method, mulberry bark fibers could be concluded. The ink used on the historical rubbings was determined as pine wood soot ink, the whole egg (egg glair and egg yolk) was used as binding media, while peppermint oil and turmeric oil were used as additives in ink.

Through the methodology chosen, maximum information with a small amount of sample could be obtained, which is important for a precious unknown sample, like historical rubbing. The material information of the

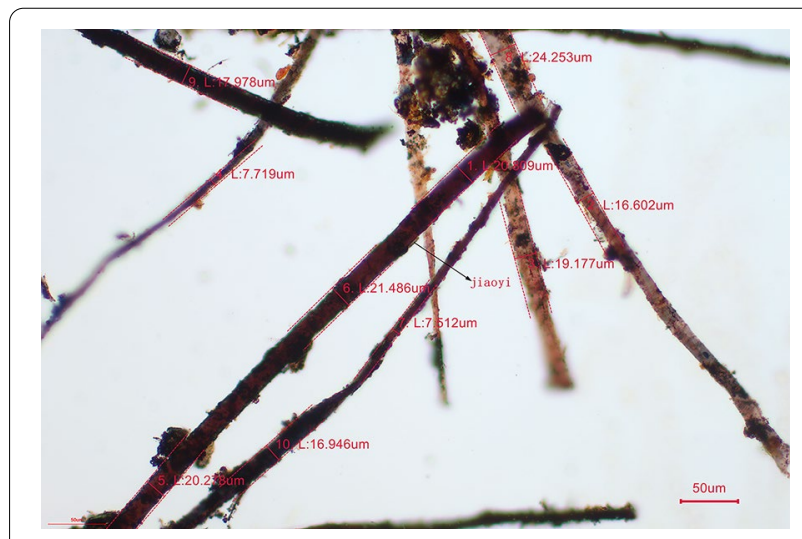

Fig. 10 The fiber morphology of historical rubbing sample $\times 20$ rubbings obtained could provide scientific support for its conservation and restoration.

\section{Abbreviations}

Py-GC/MS: Pyrolysis-Gas Chromatography/Mass Spectrometry; SEM: Scanning electron microscopy; PAHs: Polycyclic aromatic hydrocarbons.

\section{Acknowledgements \\ Not applicable.}

\section{Authors' contributions}

NY, Professor SW, and Professor QM conducted the experiments and analyzed the results. XZ supplied the precious historical rubbing sample, and provided the picture of the rubbings shown in Fig. 1. All authors reviewed the submitted article. All authors read and approved the final manuscript.

\section{Funding}

National Key Research and Development Program of China, Grant Number: 2020YFC1522402.

\section{Availability of data and materials}

The datasets used and/or analyzed during the current study are available from the corresponding author on reasonable request.

\section{Declarations}

\section{Competing interests}

The authors declare that they have no competing interests.

\section{Author details}

${ }^{1}$ Institute of Cultural Heritage and History of Science \& Technology, University of Science and Technology Beijing, Beijing 100083, PR China. ${ }^{2}$ Wuyuan Museum of Jiangxi Province, Shangrao 333299, PR China. ${ }^{3}$ Institute of Cultural Heritage, Shandong University, Qingdao 266237, PR China.

Received: 10 March 2021 Accepted: 15 September 2021

Published online: 24 September 2021

\section{References}

1. Jiang M. The making and preservation of rubbing. Archaeol Luoyang. 2018;3:79-82.

2. LiT. Pigments and fibers of ancient Chinese banknotes and pawn tickets. China Numis. 2018:1:8-18.

3. Li X, Guo J, Wang B. Research on ancient paper unearthed from the Eastern Han Dynasty tomb in Minfeng, Xinjiang. Cultural Relics. 2014;7:94-6.

4. Helman-Ważny A. More than meets the eye: fiber and paper analysis of the Chinese manuscripts from the Silk Roads. STAR Sci Technol Archaeol Res. 2016;2(2):127-40.

5. Avataneo C, Sablier M. New criteria for the characterization of traditional East Asian papers. Environ Sci Pollut Res. 2016. https://doi.org/10.1007/ s11356-016-6545-0.

6. Han B, Daheur G, Sablier M. Py-GCxGC/MS in cultural heritage studies: an illustration through analytical characterization of traditional East Asian hand-made papers. J Anal Appl Pyrol. 2016;122:458-67.

7. Han B, Vial J, Inaba M, Sablier M. Analytical characterization of East Asian handmade papers: a combined approach using Py-GCxGC/MS and multivariate analysis. J Anal Appl Pyrol. 2017;127:150-8.

8. Yao N, Wei S. Characterization and identification of traditional Chinese handmade paper via Pyrolysis-Gas Chromatography-Mass Spectrometry. BioResources. 2021;16(2):3942-51.

9. Guan L. Hai hun hou mu chu tu gu mo kao [海昏侯墓出土古墨考]. Cultural Relics World. 2020;10:69-71.

10. Wang W, Fang $X$. Zhong guo gu dai song yan mo zhi zuo gong yi yuan liu [study on the manufacturing technology of pine wood soot ink in ancient China]. Publ Print. 2010;1:21-5. 
11. Zhang W, Liu H, Guo S. A study of the manufacture and conservation of an cient ink-stick. Sci Conserv Archaeol. 1995;7(1):21-7.

12. Wang $W$, Fang $X$. Zhong guo gu dai zhi mo yu zhong yao guan xi de chu bu yan jiu [a preliminary study on the relationship between ancient Chinese ink making and traditional Chinese medicine]. Chin Mod Med. 2010;17(16):12-4.

13. Zhang $\mathrm{H}, \mathrm{Yu} \mathrm{H}$, Tang $Y$, Yang $\mathrm{G}$. Characterization of pine wood soot and lamp soot in surface chemistry. Sci Conserv Archaeol. 2018;30(1):91-9.

14. Cao $X$, Yang J, Fang $X$, Wang $C$. The Study of ink sticks excavated from Leitiao Tomb in Nanchang, Jiangxi province. Cultural Relics South Chin. 2011;2:154-7.

15. Wei S, Fang X, Cao X, Schreiner M. Characterization of the materials used in Chinese ink sticks by pyrolysis-gas chromatography-mass spectrometry. J Anal Appl Pyrol. 2011;91:147-53.

16. Wei S, Fang X, Yang J, Cao X, Pintus V, Schreiner M, Song G. Identification of the materials used in an Eastern Jin Chinese ink stick. J Cult Herit. 2012;13:448-52.

17. Ren M, Wang R, Yang Y. Identification of the proto-inkstone by organic residue analysis: a case study from the Changle Cemetery in China. Herit Sci. 2018;6:19

18. Li T. Indigo and Madder Dyed 11th to 13th century Chinese papers unearthed at Khara Khoto in Northwestern China. Tangut Res. 2017;3:3-14.
19. Chiavari G, Galletti GC, Lanterna G, Mazzeo R. The potential of pyrolysisgas chromatography/mass spectrometry in the recognition of ancient painting media. J Anal Appl Pyrol. 1993;24:227-42.

20. Wang N, Gu A, Min J, Li G, Lei Y. Identification of protein binding media used in Chinese cultural relics by Pyrolysis-Gas Chromatography/Mass Spectrometry. Chin J Anal Chem. 2020;48(1):90-6. https://doi.org/10. 19756/j.issn.0253-3820.191355.

21. Guo X. Bo he you hua xue cheng fen fen xi [analysis of chemical constituents of peppermint oil]. Spec Econ Anim Plant. 2020;23(9):33-5.

22. Yan H, Li P, Zhou G, Guo S, Wu Q, Huang S, Wang G. Quality evaluation of different origins and specifications of zingiberis rhizoma medicinal materials and decoction pieces. J Chin Med Mater. 2020;43(4):817-23.

23. Qiang Y, Wei H, Fang L, Wu M, Tan S, Zeng S, Fu W. Analysis of chemical components of volatile oil in turmeric (Curcuma longa L.) from Fujian by HS-SPME-GC-MS. Chin Food Addit. 2020;31(1):147-53.

24. Wang J, Li Y. Cong ji zhong han zhi de fen xi jian ding shi lun wo guo zao zhi shu de fa ming [analysis and identification of several Han papers and Cailun's historical achievements]. Cultural Relics. 1980;26(1):80-4.

\section{Publisher's Note}

Springer Nature remains neutral with regard to jurisdictional claims in published maps and institutional affiliations.

\section{Submit your manuscript to a SpringerOpen ${ }^{\circ}$ journal and benefit from:}

- Convenient online submission

- Rigorous peer review

- Open access: articles freely available online

- High visibility within the field

- Retaining the copyright to your article

Submit your next manuscript at $\boldsymbol{\nabla}$ springeropen.com 